\title{
Recognizing Pitfalls in Breast Cancer Follow-Up: A Necessity
}

\author{
Behnam Dalfardi
}

Published online: 16 September 2014

(C) Société Internationale de Chirurgie 2014

\section{Dear Editor}

I was intrigued by the paper by Taib et al. published in the July 2014 issue of The World Journal of Surgery [1]. The authors, by introducing two models for the reasons of delayed breast cancer diagnosis, tried to explain why women present with advanced form of this malignancy and resist treatment. Such a survey can be a nice start point for further and supplementary studies in the area of breast cancer.

Undoubtedly, the management of patients with breast cancer is not limited to their initial therapy with surgery, chemotherapy or radiotherapy. The probability of various problems associated with breast malignancies (like recurrence, distant metastasis, complications resulting from surgery, chemotherapy, and radiotherapy, etc.), remind the fact that the follow-up of such patients is also important.

As seen in the diagnosis and management of cases with primary breast cancer, there may be some pitfalls in the follow-up of these patients in clinical practice. In fact, similar to the guidelines for diagnosing and managing primary breast malignancies, the proper application of those guidelines introduced for follow-up of these cases after primary treatment is not guaranteed. Probably, this will be more problematic in low-resource parts of the world.

Recognizing the pitfalls of the follow-up and knowing their causes will provide the opportunity to purposefully deal with them, and consequently, will allow breast cancer patients to have an improved quality of life after primary treatment. Therefore, it would be a good recommendation for further research in the area of breast cancer.

Conflicts of Interest None.

\section{References:}

1. Taib NA, Yip CH, Low WY (2014) A grounded explanation of why women present with advanced breast cancer. World J Surg 38:1676-1684

B. Dalfardi $(\bowtie)$

Student Research Committee, Shiraz University of Medical

Sciences, Shiraz, Iran

e-mail: Dalfardibeh@gmail.com 